\title{
Astaxanthin effectiveness in preventing multiple sclerosis in animal model
}

\author{
Bidaran $\mathrm{S}^{1}$, Ahmadi $\mathrm{AR}^{2}$, Yaghmaei $\mathrm{P}^{1}$, Sanati $\mathrm{MH}^{3}$, Ebrahim-Habibi $\mathrm{A}^{4}$ \\ Department of Biomedical Sciences, Women Research Center, Alzahra University, Tehran, Iran. \\ aahmadi@alzahra.ac.ir
}

\begin{abstract}
OBJECTIVE: The aim of the present study was to reveal the effect of therapeutic and prophylactic potential of astaxanthin in experimental autoimmune encephalomyelitis (EAE) as an acceptable model for the study of multiple sclerosis (MS).

BACKGROUND: Astaxanthin has powerful antioxidant activities as well as several essential biological functions while multiple sclerosis prevention is highly regarded by researchers.

METHODS: The astaxanthin potential in prevention of multiple sclerosis was examined in the chronic model of experimental autoimmune encephalomyelitis (EAE) by using female C57BL/6 mice induced with oligodendrocyte glycoprotein (MOG). Splenocytes were assessed to measure the levels of proinflammatory and antiinflammatory cytokines, proliferation rate and FoxP3+Treg cell frequency. Immunohistochemical examinations were performed on spinal cord and brain tissue.

RESULTS: Astaxanthin reduced splenocytes proliferation index and proinflammatory cytokine levels, and vice versa increased the anti-inflammatory cytokine levels. Immunohistochemical studies of the spinal cord and brain showed that the infiltration with inflammatory cells was highly confined in the central nervous system. Protective effects of astaxanthin were visible by assigning low score recording in clinical behavior and disease severity. CONCLUSION: Astaxanthin is a powerful tool for intervention in EAE on a model of multiple sclerosis, so it can be studied further to prevent and treat MS (Tab. 2, Fig. 3, Ref. 41). Text in PDF www.elis.sk.

KEY WORDS: astaxanthin, experimental autoimmune encephalomyelitis, female C57BL/6 mice, Haematococcus pluvialis, multiple sclerosis.
\end{abstract}

\section{Introduction}

Multiple sclerosis is a chronic inflammatory disease that is characterized by demyelination of nerves and is thought to be mediated by myelin-specific T cells (1). Experimental autoimmune encephalomyelitis (EAE) is an acceptable model for the study of MS which is induced by immunization with myelin antigens or by adoptive transfer of myelin-specific $\mathrm{T}$ cells (2). Investigating changes in proinflammatory and anti-inflammatory cytokines over time of disease progression have led to an insight into the pathogenesis of MS (3).

Astaxanthin is a xanthophyll carotenoid, found in Haematococcus pluvialis. Astaxanthin has a powerful antioxidant activity and several essential biological functions such as protection against inflammation, diabetes, cancers, cardiovascular, gastrointestinal

\footnotetext{
${ }^{1}$ Department of Biology, Science and Research Branch, Islamic Azad University, Tehran, Iran, ${ }^{2}$ Department of Biomedical Sciences, Women Research Center, Alzahra University, Tehran, Iran, ${ }^{3}$ Medical Genetics Department, National Institute for Genetic Engineering and Biotechnology, Tehran, Iran. Molecular Modeling Group, Endocrinology and Metabolism Research Institute, Tehran, and ${ }^{4}$ University of Medical Sciences (TUMS), Tehran, Iran

Address for correspondence: A. R. Ahmadi, Dr, Department of Biomedical Sciences, Women Research Center, Alzahra University, Tehran, Iran. Phone/Fax: +98.21.88049809
}

and neurological disorders, aging and age-related disease (4). Astaxanthin inhibits reactive oxygen species (ROS) and protects against free radical damage to preserve immune-system function. Astaxanthin inhibits lipid peroxidation and has several-fold greater free radical antioxidant activity than $\beta$-carotene does (5). The antioxidant properties of astaxanthin are powerful owing to its molecular structure. At the center of the astaxanthin molecule is a conjugated polyene chain and on ionone rings there are hydroxy and keto groups. Polyene chain of astaxanthin traps the free radicals in the cell membrane and its terminal ring removes the free radicals from the outside and inside of the cell membrane (6).

Disease prevention is more consequential than treatment; therefore, the objective of this study was to assess the potential of astaxanthin in protection and prophylaxis of multiple sclerosis on an animal model of EAE.

\section{Materials and methods}

This study was carried out at the Department of Biomedical Sciences, Alzahra University, Tehran, and Department of Biological Sciences, Islamic Azad University, Sciences and Research Branch. The astaxanthin extract from Haematococcus pluvialis was kindly provided by DepArtment of Biomedical Sciences, Alzahra University, Tehran, IRAN. 


\section{Astaxanthin}

Astaxanthin extraction was performed by Sarada et al method 2006 (7) and its purification was done by tThin-layer chromatography (TLC) and column chromatography after spectrophotometric assay; the purified astaxanthin was analyzed by HPLC (8).

\section{Experimental animals}

Specific pathogen-free grade, 6-8 week-old female C57BL/6 mice with average weight of $17.62 \pm .46 \mathrm{~g}$ were purchased from Pasteur Institute of Tehran/Iran. The experiments were approved by the Ethics Committee of Azad University. Mice were housed under constant environmental conditions at $\left(23 \pm 1{ }^{\circ} \mathrm{C}\right)$ and 12 -h light/dark cycle. The mice were randomly assigned to four groups (8 mice/group) consisted of: 1) Normal control group without EAE induction, 2) EAE group with untreated EAE mice, 3) 3wAXTEAE group, in which astaxanthin pretreatment started 3 weeks before EAE induction, 4) 1wAXT-EAE group in which astaxanthin pretreatment started one week before EAE induction.

The normal control and EAE groups were fed with normal diet (Pars Co., Tehran, Iran); and experimental groups were fed with pellet with oral concentration of astaxanthin for 5 weeks after immunization with MOG (0.4\% in chow, approximately $400 \mathrm{mg} /$ $\mathrm{kg}$ /day and the pellets were impregnated with astaxanthin) $(9,10)$.

\section{Immunization}

Mice were immunized subcutaneously in the flanks with 100 $\mu \mathrm{g}$ of myelin oligodendrocyte glycoprotein $\left(\mathrm{MOG}_{35-55}\right)$ peptide (MEVGWYRSPFSRVVHLYRNGK; Peptron, South Korea) emulsified in complete Freund's adjuvant (CFA) containing $400 \mu \mathrm{g}$ Mycobacterium tuberculosis (strain H37Ra, ATTC25177). Then mice received $400 \mathrm{ng}$ of pertussis toxin by intraperitoneal (ip) injection on the same day and 48 hours later.

\section{Behavioral tests}

The body weights and neurological function scores of mice were recorded daily during the experimental period. Clinical scores of EAE were assessed in double-blind way on a scale of 0 to 7 according to the following criteria: 0 : no disease; 1 : loss of tail tonicity; 2: tail paralysis; 3 : hind limb failure; 4 : hind limb paralysis; 5 : paralysis of both hind limbs; 6 : hind limbs and forelimb paralysis; 7: death (11).

\section{Evaluation of cytokines}

Thirty-five days following the induction of EAE, the animals were euthanized by a lethal dose of ketamine and xylazine (ip), then splenocytes were isolated aseptically from mice according to the methods previously described (12) and suspended in medium containing RPMI 1640 medium supplemented with $10 \%$ fetal calf serum according to the standard protocol. Red blood cells were excluded by lysis buffer. Then splenocytes $\left(2 \times 10^{6} \mathrm{cells} / \mathrm{ml}\right)$ were cultured in 24-well plates in the presence of MOG $(50 \mu \mathrm{g} / \mathrm{ml})$, and the culture supernatants were collected after $72 \mathrm{~h}$. Concentrations of cytokines IFN- $\gamma$, IL-6, IL-10 and IL-17 were measured by using ELISA kits (eBioscience, Inc., USA). The manufacturer's protocol was followed for each kit.

\section{Splenocytes proliferation assay}

By MTT assay, cell proliferation was quantified. The splenocytes were cultured in 96-well plates in RPMI 1640 medium supplemented with $10 \%$ fetal calf serum $\left(1 \times 10^{5}\right.$ cells $/ 100 \mathrm{ml} /$ well $)$ in the presence or absence of MOG35 $5_{-55}(50 \mu \mathrm{g} / \mathrm{ml})$ and after $72 \mathrm{~h}$ of incubation, cell proliferation was measured by MTT solution. Four hours prior to the end of culture, $20 \mu 1$ of MTT solution was added. DMSO was added into each well and shaken gently until formazan crystal became solubilized and optical density was estimated by measuring the absorbance at $\lambda_{\max }(550 \mathrm{~nm})$ with a micro plate reader (Bio Tek, ELX800, USA). The proliferation index was accurately calculated. The proliferation index is a ratio of $\mathrm{OD}_{550}$ of stimulated cells in the presence of MOG to $\mathrm{OD}_{550}$ of non-stimulated cells in the absence of MOG. Cell viability was investigated by trypan blue rejection test (13).

\section{FoxP $3^{+}$Treg analysis by flow cytometry}

Regulatory $\mathrm{T}$ cells were assessed by using flow cytometry kit (eBioscience, Inc., USA) according to the methods previously described (14). The manufacturer's protocol was followed for FoxP $3^{+}$Treg analysis. Suspensions of splenocytes were prepared $\left(1 \times 10^{6}\right.$ cells $\left./ 100 \mu \mathrm{l}\right)$, and immediately, FoxP $3^{+} \mathrm{CD} 4{ }^{+} \mathrm{CD} 25^{+}$regulatory $\mathrm{T}$ cells (FoxP3 ${ }^{+} \mathrm{Treg}$ ) were stained for $30 \mathrm{~min}$ at $4^{\circ} \mathrm{C}$; then percentages of $\mathrm{CD} 4{ }^{+} \mathrm{CD} 25^{+} \mathrm{Foxp} 3^{+}$cells were analyzed with a flow cytometry software package (Partec, Germany) using Cyflogic software (version: 1.2.1). Each plot exhibits mixed cells from 8 mice.

\section{Immunohistochemical studies}

The spinal cord and the brain were quickly removed from mice sacrificed by high dosage of ketamine and xylazine, and fixed in $10 \%$ buffered formalin. After embedding in paraffin, paraffin wax was removed from 5- $\mu \mathrm{m}$ thick sections in xylene after 15-min rehydration of the tissue with $3 \% \mathrm{H}_{2} \mathrm{O}_{2}$, and then washed out 3 times in PBS containing $30 \%$ sucrose at $4{ }^{\circ} \mathrm{C}$ for $5 \mathrm{~min}$. The slices were pre-treated using the method of heat-induced epitope retrieval (HIER) with sodium citrate buffer (pH 6) and 1 mM EDTA buffer (pH 8) for $20 \mathrm{~min}$, washed out 3 times with PBS. Then the slices were incubated with special antibodies against cell surface molecules for CD4 (eBioscience, Inc., USA) and CD8 (eBioscience, Inc., USA), and then washed out with PBS. Then the slices were mounted after being dehydrated. All sections were assessed for lymphocyte inflammation. To determine the degree of inflammation, the infiltrated cells (CD8+ and CD4+ T cells) in the brain and spinal cord were counted. The examiner blinded to group assignment counted the total number of CD8+ and CD4+ T cells infiltrates in brain and spinal cord sections. To count infiltrating cells in the brain and spinal cord tissue, two sections from each mouse were investigated by light microscope while the infiltrating mononuclear cells population was calculated per $1 \mathrm{~mm}^{2}$ area (15). Light images were captured with an Olympus BX51 microscope, with Olympus DP70 camera, using Olysia Bioreport software version 3.2.

\section{Statistical analysis}

Data were expressed as mean \pm S.E.M and analyzed with SPSS Version 21. Analyses were performed for three independent experi- 


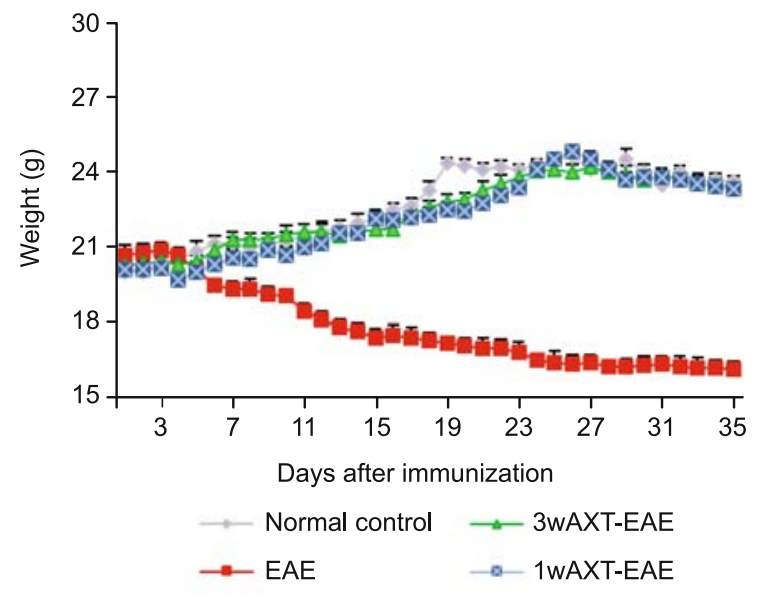

Fig. 1. Linear curve of mean changes in body weight of EAE mice and mice pretreated with astaxanthin. The mean body weight of mice in EAE significantly reduced compared with normal control group $(\mathrm{p}<$ 0.0001). The mean body weight of 3 wAXT-EAE and 1wAXT-EAE mice significantly increased compared to EAE group (8 mice per group) (p $<0.0001)(* * *$ A significant difference compared with EAE group).

ments. Analysis of variance for comparison of clinical parameters (two-way ANOVA) and parameters such as cytokines, splenocytes proliferation and FoxP $3{ }^{+}$Treg cells analysis (one-way ANOVA), and nonparametric data (The mean body weight and clinical score) were compared by Mann-Whitney U test. Statistical $\mathrm{p}<0.05$ were regarded as a significant level.

\section{Results}

\section{Clinical evaluation}

Following the induction of EAE, the mice were examined every day for clinical disease symptoms and mortality, and their body

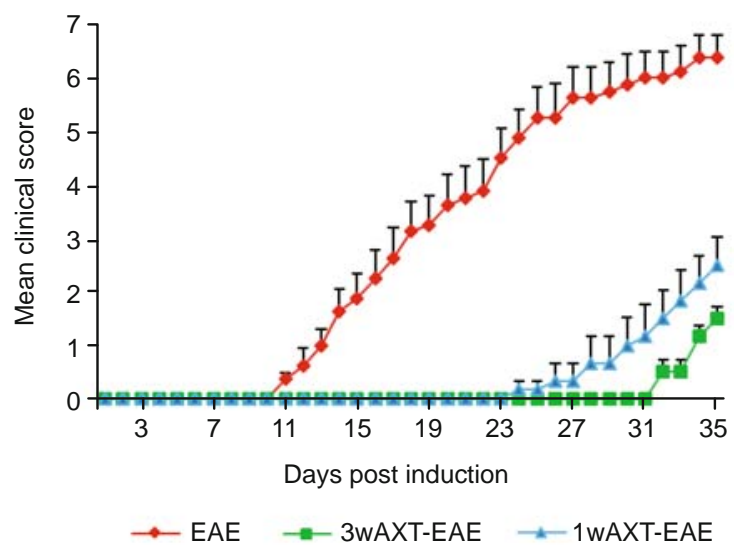

Fig. 2. Effects of astaxanthin on clinical signs of EAE: The mean clinical score of EAE in the 3wAXT-EAE and 1wAXT-EAE significantly reduced in comparison to the EAE group $(* * * p<0.0001)$. Also the first clinical score as clinical symptom was shown in EAE and the 3wAXTEAE and 1wAXT-EAE groups on the 11th and 32th and 24th day post-immunization respectively. In fact, the onset of the first clinical symptom in the 3wAXT-EAE and 1WAXT-EAE are delayed compared with the EAE.The mean clinical score of EAE in the 1wAXT-EAE significantly increased comparison to the 3wAXT-EAE $(\# p<0.0001)$.

weight was also measured. The mean body weight of EAE group significantly decreased compared to normal control $(p<0.05)$. This weight loss occurred six days after immunization. But the mean body weight of 3wAXT-EAE and 1wAXT-EAE groups increased significantly in comparison with EAE $(\mathrm{p}<0.0001)$ (Fig. 1).

The mean clinical score of EAE in 3wAXT-EAE and 1wAXTEAE groups were significantly $(p<0.0001)$ lower than those in EAE group (Fig. 2) while in both prevention groups, the clinical signs were less severe and mice had a later onset of the clinical signs compared with EAE group.

Tab. 1. Effects of Astaxanthin pretreatment on splenocytes proliferation index and levels of cytokine production and frequency of CD4+CD25+Foxp3+ $T$ cells in splenocytes. Proliferation index and cytokine levels were measured in splenocytes from mice of the control, non-treated-EAE and two experimental groups on day 35 post immunization. Astaxanthin significantly reduced IFN- $\gamma$, IL-6 and IL-17 levels on the 3wAXT-EAE and 1wAXT-EAE groups compared to EAE group and the level of IFN- $\gamma$ in the 3wAXT-EAE group showed a significant decrease compared to 1wAXT-EAE group, but IL-6 and IL-17 level in 3wAXT-EAE group compared to 1wAXT-EAE group had no significant difference. Astaxanthin increased IL-10 level significantly in 3wAXT-EAE and 1wAXT-EAE geoupa compared with EAE group. In contrast, IL-10 in EAE group decreased significantly in comparison with the normal control group, and IL-10 level was significantly reduced in 1wAXT-EAE group compared to the normal control group. There was also a significant difference in IL-10 level in 3wAXT-EAE group. The level of IL-10 in 3wAXTEAE group showed a significant increase compared to 1wAXT-EAE group. Astaxanthin inhibited the EAE-associated proliferation. MTT test results showed decreases in lymphocyte proliferation in 3wAXT-EAE and 1wAXT-EAE groups compared to mice with EAE. The percentage of $\mathrm{CD4}^{+} \mathrm{CD}^{2}{ }^{+} \mathrm{Foxp}^{+} \mathrm{T}$ cells was determined on day 35 post immunization by flow cytometric analysis. The population of FoxP3 ${ }^{+} \mathrm{Treg}$ cells rose in splenocytes derived from mice from 3 wAXT-EAE and 1wAXT-EAE groups. FoxP3 ${ }^{+}$Treg cells in 3 wAXT-EAE and 1wAXT-EAE groups showed a significant increase compared to EAE. But there was no significant difference between the two experimental groups.

\begin{tabular}{|c|c|c|c|c|}
\hline Test - Groups & Normal Control & EAE & 3wAXT-EAE & 1wAXT-EAE \\
\hline $\mathrm{IFN} \gamma(\mathrm{pg} / \mathrm{ml})$ & $13.53 \pm 1.87$ & $816.77 \pm 14.44 \dagger \dagger \dagger$ & $83.3 \pm 3.43 * / \dagger \dagger$ & $118.93 \pm 2.26 * * * / \dagger \dagger / \#$ \\
\hline IL_6 (pg/ml) & $30 \pm 2.14$ & $280.83 \pm .64 \dagger \dagger$ & $111.8 \pm 3.35 * / \uparrow$ & $139.67 \pm 3.99 * / \uparrow \dagger \dagger$ \\
\hline IL_10 (pg/ml) & $81.01 \pm 0.486$ & $25.2 \pm 1.46 \dagger \dagger$ & $78.63 \pm 1.88 *$ & $65.9 \pm 2.85 * / \uparrow / \#$ \\
\hline IL_17 (pg/ml) & $8.4 \pm 0.83$ & $411.67 \pm 10.93 \dagger \dagger$ & $65.3 \pm 3.52 * / \uparrow$ & $80.17 \pm 2.68 * * / \uparrow \dagger$ \\
\hline Proliferation Index (MTT test) & - & $1.812 \pm 0.012$ & $0.9035 \pm 0.014 * *$ & $1.03 \pm 0.01 *$ \\
\hline Frequency of CD4+CD25+Foxp3+T cells (\%) & $0.94 \pm 0.006$ & $1.94 \pm 0.32$ & $5.16 \pm 0.012 * * *$ & $3.84 \pm 0.023 *$ \\
\hline
\end{tabular}

Results were expressed as the mean \pm SEM from 8 mice/ group. $* \mathrm{p}<0.05$ and $* * \mathrm{p}<0.001$ and $* * * \mathrm{p}<0.0001$ compared with untreated EAE $(*$ versus EAE group, \# versus the 3wAXT-EAE group, $\dagger$ versus normal control group). 

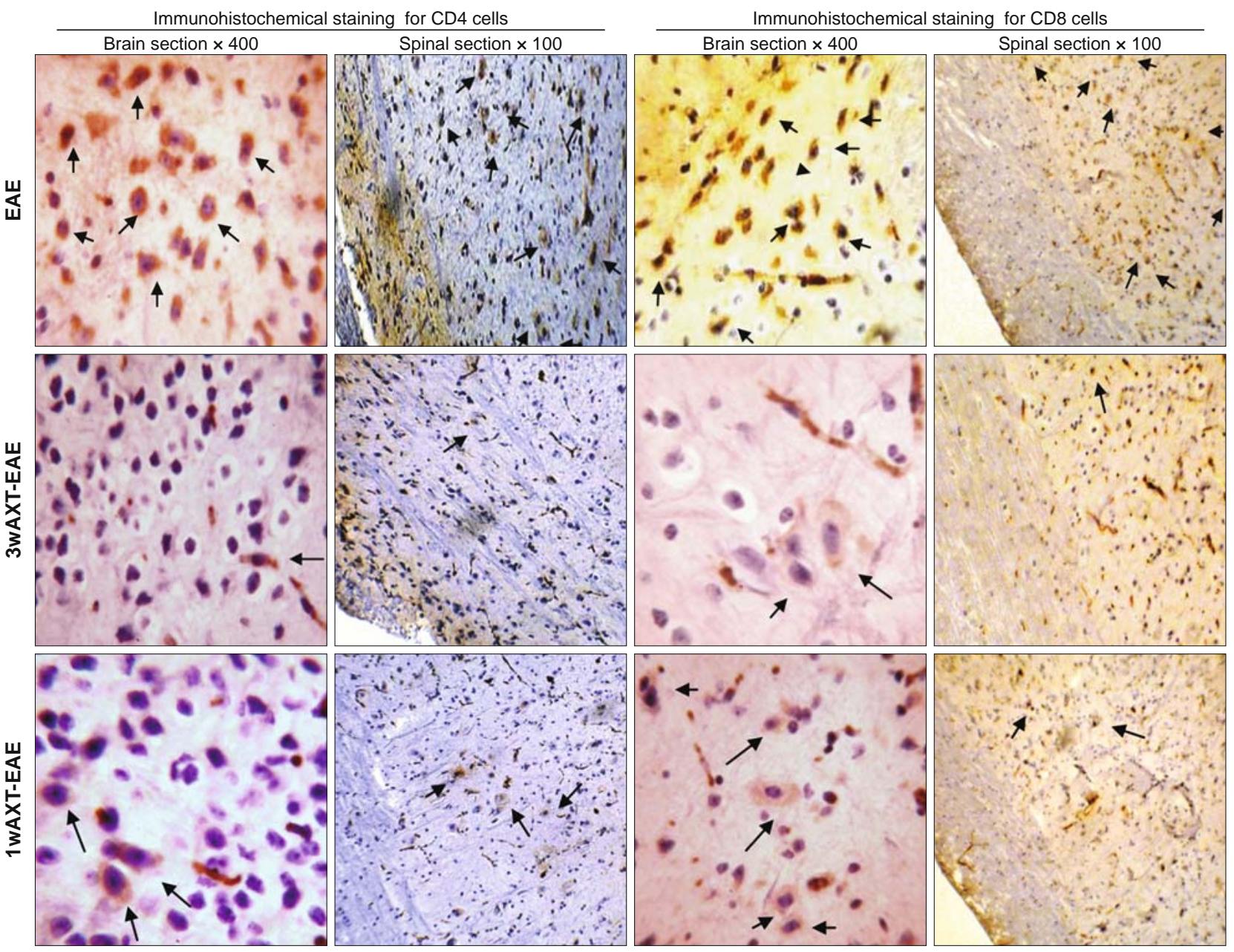

Fig. 3. Immunohistochemical studies of the effects of astaxanthin pretreatment: astaxanthin pretreatment reduced EAE-induced immune cell infiltration into the spinal cord and the brain in female C57BL/6 mice. The images above indicate immunohistochemical staining of the spinal cord and the brain with CD4 antibody and CD8 antibody against cell surface CD4 and CD8 molecules. Immunochemically stained showed that subsets of lymphocytes labeled infiltrated into the spinal cord and brain of EAE mice. But astaxanthin pretreatment reduced the density of CD4 and CD8 in the spinal cord and brain of 3wAXT-EAE and 1wAXT-EAE compared to the EAE mice (arrows).

The onset of clinical symptoms after immunization in EAE, 3wAXT-EAE and 1wAXT-EAE groups was observed on days 11, 32 and 24, respectively (Fig. 2).

\section{Cell proliferation and cytokine production analysis}

EAE was associated with proliferation. In this study, by MTT assay, we observed that astaxanthin inhibited the EAE-associated proliferation in 3wAXT-EAE and 1wAXT-EAE groups compared to EAE group $(p<0.0001)$ (Tab. 1). The results of assessment of proinflammatory and anti-inflammatory cytokines of splenocytes revealed the severity of disease and recovery rate in EAE and experimental groups. The results showed that astaxanthin significantly reduced the levels of IFN- $\gamma$, IL- 6 and IL-17 in 3wAXT-EAE and 1wAXT-EAE groups $(\mathrm{p}<0.0001)$ compared with EAE group, and astaxanthin increased the level of IL-10 in 3wAXT-EAE and 1wAXT-EAE groups compared with EAE group $(\mathrm{p}<0.0001)$ (Tab. 1). As shown in Table 1, IFN- $\gamma$, IL-6, IL-17 and IL-10 levels in the experimental groups are significantly different from those in the normal control group.

\section{Quantitative definition of Foxp3 ${ }^{+}$T Cells}

Our research findings showed that pretreatment with astaxanthin significantly increased FoxP ${ }^{+}$Treg cells in splenocytes derived from mice from 3wAXT-EAE and 1wAXT-EAE groups compared to those from EAE mice $(p<0.0001$ and $p<0.05$, respectively) (Tab. 1).

Immunohistochemical studies of the effects of astaxanthin, namely the reduction of infiltration with immune cells in the spinal cord and brain of pre-treated EAE mice

The spinal cord and brain sections were stained immuno-histochemically with CD4 and CD8 (Fig. 3) which were antibodies against cell surface molecules and it became clear that CD4 and CD8 immuno-reactivity increased in the spinal cord and brain of 
Tab. 2. Result of immunohistochemical studies of the effects of astaxanthin pretreatment: Astaxanthin pretreatment reduced the density of CD4 and CD8 in the spinal cord and brain in 3wAXT-EAE group and 1wAXT-EAE compared to the EAE mice $(p<0.0001)$. Leukocyte infiltration (including both $\mathrm{CD}^{+}$and $\mathrm{CD}^{+} \mathrm{T}$ cells) into the spinal cord and brain of mice from 1wAXT-EAE group were increased compared with 3wAXT-EAE mice $(p<0.001)$. Data were presented by mean \pm SEM of 8 mice (*versus EAE group and \#versus 3wAXT-EAE).

\begin{tabular}{lccccc}
\hline & \multicolumn{3}{c}{ Brain } & & \multicolumn{2}{c}{ spinal } \\
\cline { 2 - 3 } \cline { 5 - 6 } & $\mathrm{CD} 4{ }^{+}$cells $/ \mathrm{mm}^{2}$ & $\mathrm{CD} 8^{+}$cells $/ \mathrm{mm}^{2}$ & & $\mathrm{CD} 4^{+}$cells $/ \mathrm{mm}^{2}$ & CD8 $8^{+}$cells $/ \mathrm{mm}^{2}$ \\
\hline EAE & $35.63 \pm 4.07$ & $20.25 \pm 1.16$ & & $15.38 \pm 1.32$ & $12 \pm 1.07$ \\
\hline 3WAXT-EAE & $3.5 \pm 0.57 * * *$ & $2.0 \pm 0.38^{* * *}$ & & $6.25 \pm 0.75^{* * *}$ & $2 \pm 0.38^{* * *}$ \\
\hline 1WAXT-EAE & $11.125 \pm 1.27 * * * / \# \#$ & $3.25 \pm 0.37 * * *$ & & $8.25 \pm 0.73^{* * *}$ & $7.13 \pm 0.51^{* * *} / \# \# \#$ \\
\hline
\end{tabular}

EAE mice. Microscopic images revealed a sporadic presence of CD4 and CD8 in the spinal cord and brain of EAE mice pretreated with astaxanthin in 3wAXT-EAE and 1wAXT-EAE groups, and it was clearly seen that their density is lower compared to EAE. The spinal cord and brain sections obtained from EAE mice showed infiltration of mononuclear cells around the vessels of the spinal cord and brain. However, in 3wAXT-EAE and 1wAXT-EAE groups, the mononuclear infiltration into the spinal cord and brain was lowered ( $p<0.0001)$ (Fig. 3, Tab. 2).

\section{Discussion}

In this study, we have observed that the prophylactic use of astaxanthin provides protection against the development of EAE as studied on MS model. Also, the protective role of astaxanthin was manifested at clinical, histological and molecular levels.

Astaxanthin consumption as a pre-treatment delayed the onset and decreased the severity of the disease, and detracted immune cell infiltration into white matter of spinal cord and brain. Study of the structure of astaxanthin can explain its antioxidative, antiinflammatory and immune-modulatory activities. Astaxanthin is able to pass through the blood-brain barrier (BBB) and tighten the cell membrane (7). Structurally, the astaxanthin molecule has hydroxyl- and keto groups, which are at the ends of the molecule (16) and a conjugated polyene chain at the center of molecule. Hydroxyl groups span the lipid bilayer and are placed close to the lipid membrane fluid phase, which as a result, leads to a decrease in membrane fluidity and maintains the structure and function of membrane (17). Astaxanthin restricts the penetration of lipoperoxidation promoters in the cell membrane, and inhibits lipid peroxidation (18).

EAE is the failure of BBB, which leads to the inflammation of nervous tissue while during inflammation, the permeability of $\mathrm{BBB}$ increases. The presence of astaxanthin due to its transgression through BBB before EAE induction strengthens cell membranes. As a result, the infiltration of immune cells into CNS is reduced. Moreover, it has been shown in other studies that astaxanthin protects cells against oxidative damage through various mechanisms by removing singlet oxygen, inhibiting lipid peroxidation and regulating gene expression related to oxidative stress $(19,20)$.

Astaxanthin establishes the BBB integrity by affecting the regulation of inflammatory cytokines, while cytokines as signaling proteins, play major roles in the regulation of immune responses in EAE (21). In this study, we observed that astaxanthin signifi- cantly reduced the splenocytes inflammatory parameters such as IFN- $\gamma$, IL-6, IL-17, and increased anti-inflammatory cytokines such as IL-10.

Th1 cytokines associate in the progression of disease while Th2 cytokines play an important role in the recovery of EAE-mice (22). Therefore, Th2 cells can control the function and development of Th1 cells in EAE (23).

In the study of the therapeutic effects of astaxanthin on Helicobacter pylori-infected mice suggested that astaxanthin eliminates free radicals and can modify the immune response to $H$. pylori with shifting the Th1 response to Th2 cell response (24). The immune response caused by the Th1 cell-mediated response leads to the release of IFN- $\gamma$, which activates phagocytic cells and is effective in repairing the mucosal damage (25).

The results of the in vitro study showed that astaxanthin modulates the production of Th1 cytokines and regulates lymphocytic immune responses (26). In this study we found out that the production of Th1 cytokines such as IFN- $\gamma$ was reduced in experimental groups. Astaxanthin modifies the immune response by shifting the Th1 to Th2 cell response, and leads to increased production of IL-10 level. The production of IL-6 and IL-17 is involved in MS development (27). IL-17, by degradation of BBB, causes demyelination and neuroinflammation (28). Astaxanthin by inhibiting ROS, can reduce inflammatory mediators in arthritis (29).

In this study, IL-6 and IL-17 levels were reduced in 3wASTEAE and 1wAST-EAE groups compared to EAE. This result may reflect the effect of astaxanthin of maintaining the integrity of $\mathrm{BBB}$, which reduces inflammation. Astaxanthin's ability to downregulate MOG35-55-specific lymphocyte proliferation reduces the number of potentially encephalitogenic T lymphocytes, which is a major factor in the pathogenesis of EAE (26). In this study, the cell proliferation has also diminished significantly in the experimental groups compared to EAE-mice.

Regulatory $\mathrm{T}$ cells as $\mathrm{CD} 4{ }^{+} \mathrm{CD} 25^{+} \mathrm{Foxp} 3^{+}$Treg cells have a specific role in creating the balance between immunity and tolerance. Since MS is an inflammatory autoimmune disease, Treg cells are thought to be involved in MS pathogenesis (30) Reducing the number of Treg cells leads to severe autoimmune diseases (31).

In the study ahead, the Treg cells' numbers were increased in the experimental groups that had used astaxanthin 1 or 3 weeks before immunization. Also, our observations from the immunohistochemical analysis show that astaxanthin consumption in the period prior to immunization alleviates the inflammatory infiltrates in the spinal cord and brain. Immunohistochemistry of the spinal 
cord and brain with antibodies specific for cell surface molecules for CD4 and CD8 indicate that immunoreactivities of CD4, CD8 become reduced in the experimental prevention groups compared with EAE.

Astaxanthin applies antioxidant activity through various mechanisms such as absorption of free radicals into the polyene chain, formation of chemical bonds with reactive species or electron donation (4). Reports showed that astaxanthin interferes with the growth and differentiation of the nerve cell. Possibly, astaxanthin affects the cell growth or differentiation and neurogenesis by modulating ERK signals. ERK signaling pathway is defined as part of the superfamily of the mitogen-activated protein kinases (MAPK). MAPKs are involved in cellular responses to a variety of stimuli such as pro-inflammatory cytokines. They regulate cell function, including proliferation, gene expression, differentiation, cell survival, and apoptosis (32).

ERK is one of the major regulating molecules involved in neurogenesis. Astaxanthin-induced activation of ERK pathway facilitates the NRF2 release, allowing the protein transports to the nucleus and increases the number of transcriptional antioxidant defense mechanisms such as HO- 1 . The upregulation of HO-1 is assigned to protect cells against oxidative stress resulting from exposure to beta-amyloid, representing another mechanism through which astaxanthin can decrease oxidative stress and protect the CNS (33).

Astaxanthin, by activation of PI3K/Akt and ERK signaling pathways, causes the dissociation and nuclear translocation of nuclear erythroid 2-related factor (Nrf2). This powerful antioxidant has the role of a negative regulator for Sp1/NR1 signaling pathway, and decreases the production of oxidative stress (34). AKT, PI3K, and MEK are also involved in signal transmission pathways with known roles in cell growth and differentiation (35). Astaxanthin, on 1-methyl-4-phenyl-1,2,3,6-tetrahydropyridine (MPTP), induced apoptosis of the substantial nigra neurons in the mouse model of Parkinson by inhibiting MPP+ effect, and stopped production of intracellular ROS (36). For normal CNS function, energy production, and redox sensitive signaling pathways reducing the reactive oxygen species (ROS) production are essential. Under normal circumstances, the quantities of ROS are regulated by endogenous antioxidant mechanisms which act to protect against excessive ROS production and consequent tissue damage $(37,38)$.

ROS induce redox-sensitive transcription factors such as nuclear factor $\kappa \mathrm{B}(\mathrm{NF}-\kappa \mathrm{B})$ and activator protein 1 (AP-1), and these transcription factors regulate genes controlling the production of inflammatory cytokines and adhesion molecules which induce phagocytic infiltration. In return, astaxanthin inhibits ROS-induced production of these transcription factors, which leads to reduced inflammation (39). Astaxanthin suppressed ROS-induced nuclear expression of NF- $\mathrm{KB}$ and alleviated the downstream production of pro-inflammatory cytokines (40). Astaxanthin suppresses nuclear expression of NF- $\mathrm{kB}$ and reduces downstream proinflammatory cytokines production, and by suppressing I $\mathrm{B}-\alpha$ degradation and $\mathrm{NF}-\kappa \mathrm{B}$ nuclear translocation, inhibits the expression of inflammatory molecules IL-6, intercellular adhesion molecule-1 (ICAM-1), and monocyte chemoattractant protein-1(MCP1) (41).
Due to the mechanisms mentioned above, it can be concluded that astaxanthin, before and after immunization, by passing through the $\mathrm{BBB}$, protects $\mathrm{CNS}$ against chronic neurodegeneration and via reducing the production of ROS and oxidative stress, it decreases the severity of the disease.

According to our knowledge, this is the first study that evaluates the astaxanthin pre-treatment effect on the animal model of MS (EAE). As the evidence showed that consumption of astaxanthin before EAE induction has positive effects on prevention of the disease, declination of disease progression, and as a result, the behavioral symptoms diminish and subjects regain abilities.

\section{References}

1. Muller D, Pender M, Greer J. A neuropathological analysis of experimental autoimmune encephalomyelitis with predominant brain stem and cerebellar involvement and differences between active and passive induction. Acta Neuropathol 2000; 100: 174-182.

2. Bettelli E, Korn T, Oukka M, Kuchroo VK. Induction and effector functions of Th17 cells. Nature 2008; 453: 1051-1057.

3. Martins TB, Rose JW, Jaskowski TD, Wilson A, Husebye D, Seraj HS, Hill HR. Analysis of Proinflammatory and Anti-Inflammatory Cytokine Serum Concentrations in Patients With Multiple Sclerosis by Using a Multiplexed Immunoassay. Am J Clin Pathol 2011; 136: 696-704.

4. Ranga Rao A, Phang SM, Ravi S, Aswathanarayana RG. Astaxanthin: Sources, extraction, stability, biological activities and its commercial applications - A review. Mar. Drugs 2014; 12: 128-152.

5. Pashkow FJ, Watumull DG, Campbell CL. Astaxanthin: A novel potential treatment for oxidative stress and inflammation in cardiovascular disease. Am J Cardiol 2008; 101: 58D-68D.

6. Goto S, Kogure K, Abe K, Kimata Y, Kitahama K, Yamashita E, Terada H. Efficient radical trapping at the surface and inside the phospholipid membrane is responsible for highly potent antiperoxidative activity of the carotenoid astaxanthin. Biochim Biophys Acta 2001; 1512: 251-258.

7. Sarada R, Vidhyavathi R, Usha D, Ravishankar GA. An Efficient Method for Extraction of Astaxanthin from Green Alga Haematococcus pluvialis. J Agric Food Chem 2006; 54: 7585-7588.

8. Golkhoo S, Barantalab F, Ahmadi AR, Hassan ZM. Purification of Astaxanthin from mutant of Phaffia rhodozyma JH-82 which isolated from forests trees of Iran. Pak J Biol Sci 2007; 10 (5):802-805.

9. Chew BP, Park JS, Wong MW, Wong TS. A comparison of the anticancer activities of dietary beta-carotene, canthaxanthin and astaxanthin in mice in vivo. Anticancer Res 1999; 19 (3A): 1849-1853.

10. Yuri T, Oshizawa K, Emoto Y, Kinoshita Y, Yuki M et al. Effects of dietary Xanthophylls, Canthaxanthin and Astaxanthin on N-MethylN-nitrosourea-induced Rat Mammary Carcinogenesis. In vivo 2016; 30 : 795-800.

11. Mosayebi G, Ghazavi A, Salehi H, Payani MA, Khazae MR. Effect of sesame oil on the inhibition of experimental autoimmune encephalomyelitis in C57BL/6 mice. Pak J Biol Sci 2007; 10 (11): 1790-1796.

12. Ayala A, Deol ZK, Lehman DL, Herdon CD, Chaudry IH. Polymicrobial sepsis but not low dose endotoxin infusion causes decreased splenocyte IL-2/IFN-gamma release while increasing IL-4/IL-10 production. J Surg Res 1994; 56: 579-585. 


\section{0-166}

13. Ramirez CN, Antczak C, Djaballah H. Cell Viability Assessment: Toward Content-Rich Platforms. Expert Opinion Drug Discov 2010; 5 (3): $223-233$

14. Wisnoski N, Chung CS, Chen Y, Huang X, Ayala A. The contribution of CD4+ CD25+ T-regulatory-cells to immune suppression in sepsis. Shock 2007; 27 (3): 251-257.

15. Brochard V, Combadière B, Prigent A, Laouar Y, Perrin A, BerayBerthat $\mathbf{V}$ et al. Infiltration of CD4+ lymphocytes into the brain contributes to neurodegeneration in a mouse model of Parkinson disease. J Clin Invest 2009; 119 (1): 182-192.

16. Ginter E, Simko V, Panakova V. Antioxidants in health and disease. Bratisl Lek Listy 2014; 115 (10): 603 - 606.

17. Barros MP, Pinto E, Colepicolo P, Pedersen M. Astaxanthin and peridinin inhibit oxidative damage in $\mathrm{Fe} 2+$-loaded liposomes: scavenging oxyradicals or changing membrane permeability? Biochem Biophy Res Comm 2001; 288: 225-232.

18. Wisniewska A, Subczynski WK. Effects of polar carotenoids on the shape of the hydrophobic barrier of phospholipids bilayers. Biochim Biophys Acta 1998; 1368: 235-246.

19. Stahl W, Sies H. Antioxidant activity of carotenoids. Mol Asp Med 2003; 24: 345-351

20. Naito Y, Uchiyama K, Aoi W, Hasegawa G, Nakamura N et al. Prevention of diabetic nephropathy by treatment with Astaxanthin in diabetic $\mathrm{db} / \mathrm{db}$ mice. Biofactors 2004; 20: 49-59.

21. Hirano T, Yasukawa K, Harada H, Taga T, Watanabe Y, Matsuda T et al. Complementary DNA for a novel human interleukin (BSF-2) that induces B lymphocytes to produce immunoglobulin. Nature 1986; 324 (6092): 73-76.

22. Miller SD, Karpus WJ. The immunopathogenesis and regulation of Tcell-mediated demyelinating diseases. Immunol Today 1994; 15: 356361.

23. Mosmann TR, Coffman RL. Th1 and Th2 cells: different patterns of lymphokine secretion lead to different functional properties. Ann Rev Immunol 1989; 7: 145-173.

24. Akyon Y. Effect of antioxidants on the immune response of Helicobacter pylori. Clin Microbiol Infect 2002; 8: 438-441.

25. Lindholm C, Quiding-Jarbink M, Lonroth H, Hamlet A, Svernnerholm AM. Local cytokine response in Helicobacter pylori-infected subjects. Infect Immun 1998; 66: 5964-5971.

26. Kuan-Hung L, Kao-Chang L, Wan-Jung L, Philip-Aloysius T, Thanasekaran $\mathbf{J}$ et al. Astaxanthin, a Carotenoid, Stimulates Immune Responses by Enhancing IFN- and IL-2 Secretion in Primary Cultured Lymphocytes in vitro and ex vivo. Int J Mol Sci 2016; 17 (1): 1-10.

27. Graber JJ, Dhib-Jalbut S. Protective autoimmunity in the nervous system. Pharmacol Ther 2009; 121 (2): 147-159.
28. Jadidi-Niaragh F, Mirshafiey A. Th17 cell, the new player of neuroinflammatory process in multiple sclerosis. Scand J Immunol 2011; 74: 1-13.

29. Kimble LL, Mathison BD, Chew BP. Astaxanthin Mediates Inflammation Biomarkers Associated with Arthritis in Human Chondrosarcoma Cells Induced with Interleukin-1 $\beta$. Amer J Adv Food Sci Technol 2013; 2: $37-51$.

30. Kouchaki E, Salehi M, Sharif MR, Nikoueinejad H, Akbari H. Numerical status ofCD4+CD25+FoxP3+ and CD8+CD28- regulatory T cells in MS. Iran J Basic Med Sci 2014; 17: 250-255.

31. Buckner JH. Mechanisms of impaired regulation by CD4+CD25+FOXP3+regulatory T cells in human autoimmune diseases. Nat Rev Immunol 2010; 10 (12): 849-859.

32. Kim YH, Koh HK, Kim DS. Down-regulation of IL-6 production by astaxanthin via ERK-, MSK-, and NFkappaB- mediated signals in activated microglia. Int Immunopharmacol 2010; 10: 1560-1572.

33. Li Z, Dong X, Liu H, Chen X, Shi H, Fan Y, Hou D, Zhang X. Astaxanthin protects ARPE-19 cells from oxidative stress via upregulation of Nrf2-regulated phase II enzymes through activation of PI3K/Akt. Mol Vis 2013; 19: 1656-1666.

34. Wang HQ, Sun XB, Xu YX, Zhao H, Zhu QY et al. Astaxanthin upregulates heme oxygenase-1 expression through ERK1/2 pathway and its protective effect against beta-amyloid-induced cytotoxicity in SH-SY5Y cells. Brain Res 2010; 1360: 159-167.

35. Grimmig B, Kim SH, Nash K, Bickford PC, Douglas Shytle R. Neuroprotective mechanisms of astaxanthin: a potential therapeutic role in preserving cognitive function in age and neurodegeneration. GeroScience 2017; 39: 19-32.

36. Dae-Hee L, Yong JL. Astaxanthin protects against MPTP/MPP+induced mitochondrial dysfunction and ROS production in vivo and in vitro. Food Chem Toxicol 2011; 49 (1): 271-280.

37. Koutsilieri E, Scheller C, Tribl F, Riederer P. Degeneration of neuronal cells due to oxidative stress-microglial contribution. Parkinsonism Relat Disord 2002; 8: 401-406.

38. Lee D-H, Kim C-S, Lee YJ. Astaxanthin protects against MPTP/ MPP+ -induced mitochondrial dysfunction and ROS production in vivo and in vitro. Food Chem Toxicol 2011; 49: 271.

39. Aoi W, Naito Y, Sakuma K, Kuchide M, Tokuda H et al. Astaxanthin limits exercise-induced skeletal and cardiac muscle damage in mice. Antioxid Redox Signal 2003; 5: 139-144.

40. Speranza L, Pesce M, Patruno A, Franceschelli S, de Lutiis MA et al.Astaxanthin treatment reduced oxidative induced pro-inflammatory cytokines secretion in U937: SHP-1 as a novel biological target. Mar Drugs 2012; 10: 890-899.

41. Ohgami K, Shiratori K, Kotake S, Nishida T, Mizuki N et al. Effects of astaxanthin on lipopolysaccharide-induced inflammation in vitro and in vivo. Invest OphthalmolVis Sci 2003; 44: 2694-2701. 